\title{
COULD THE QUARK ELECTROWEAK AND MASS EIGENSTATES COINCIDE? $\star$ ฟ
}

\author{
F. DEL AGUILA ', G.L. KANE and M. QUIRÓS ${ }^{2}$ \\ Randall Physics Laboratory, University of Michigan, Ann Arbor, MI 48109, USA
}

Received 14 July 1987

\begin{abstract}
We conjecture that in the absence of new mixing effects at the weak scale the standard quark mass eigenstates and the quark weak eigenstates would coincide, and that for all three families a simple hierarchy would apply, with $m_{\mathrm{u}}^{0}>m_{\mathrm{d}}^{0}$. We further assume that the observed mass inequality $m_{\mathrm{d}}>m_{\mathrm{u}}$ arises from mixing of $\mathrm{d}$ with a heavier quark $\mathrm{D}$. This approach leads to a CKM matrix that is automatically expressed in terms of mass ratios and different from other ones that have been studied; it is consistent with experimental constraints. In addition to requiring the existence of $\mathrm{D}$ with a mass on the electroweak scale, in general an unambiguous prediction is that $\left|V_{\mathrm{td}}\right|=\left|V_{\mathrm{ub}}\right|$, which is soon testable. The approach can be implemented in $\mathrm{E}_{6}$-based models.
\end{abstract}

Introduction. As is frequently remarked, although the standard model [1] is a full theory and is consistent with all experiments, it is conceptually unsatisfactory in various ways. One of them is that all masses have to be put in by hand; although that can be done technically with the Higgs mechanism, the origin of mass is not understood. It may be, of course, that progress will not come until there is a complete theory. Alternatively, perhaps the pattern of measured masses will lead to at least partial insights, as has so often happened in the past when data led to theoretical developments.

With quark masses, the observed situation is not very suggestive. Even the simplest regularity, that $m_{\mathrm{t}}>m_{\mathrm{b}}$ and $m_{\mathrm{c}}>m_{\mathrm{s}}$, is violated for the lightest family. One thing that we propose here is that the reversal of the ordering is telling us something significant. Namely, we conjecture that the mass of the down-quark $d$ is shifted from the value it has in the fermion lagrangian by mixing with a heavier quark $D$ of the same color and electric charge.

The qualitative logic that leads us in this direction is that such a mixing can give a mass matrix for $\mathrm{d}$ and $\mathrm{D}$ of the form $\left(\begin{array}{cc}\delta & m \\ m & M\end{array}\right)$ where $\delta$ is small and can be ignored, and $m \ll M$. Then, as is familiar in the see-saw mechanism, the eigenvalues are $m_{2} / M, M$, so the lighter mass has a value that is uncoupled from its unmixed value if $m^{2} / M \gg \delta$. No tuning or cancellation is required. In addition, group representations with $Q=-1 / 3$ quarks that are $\mathrm{SU}(2)$ singlets have become very familiar in recent years, so we know immediately that we can implement such ideas in specific models.

Another way to view our argument is that we are assuming that the quark weak eigenstates and mass eigenstates would be identical in the absence of mixing. The mixing effectively breaks SU(2) and rotates the mass eigenstates. The d-quark with definite mass contains an SU(2) singlet piece of order $m / M$.

One consequence of this approach is that the heavier quark must have a mass of the order of one hundred to a few hundred $\mathrm{GeV}$, or the idea would not make much sense. Esthetically we find that very attractive for an anthropic reason; that $m_{\mathrm{d}}>m_{\mathrm{u}}$ is crucial for the form our world takes, since it allows neutron $\beta$-decay and affects a number of cosmological aspects of the way the world is formed. It is attractive to us to account for

* Research supported in part by the US DOE and by CAICYT.

1 Permanent address, Department de Física Teòrica, Universitat Autonoma de Barcelona, E-08193 Bellaterra (Barcelona), Spain.

2 Permanent address, CSIC, Serrano 119, E-28006 Madrid, Spain. 
such an effect in terms of simple mass regularities and physics at the electroweak scale, rather than fine tuning of feed-down effects from the Planck scale or the GUT scale [2].

As we will see in the following, pursuing this logic implies a CKM matrix [3] where the quark mixing angles are given by products of ratios of masses. The resulting expressions for the elements of the CKM matrix will give in general one prediction, $\left|V_{\mathrm{td}}\right|=\left|V_{\mathrm{ub}}\right|$, without further assumptions. Since the size of the recently observed $\mathrm{B}_{\mathrm{d}}^{0}-\overline{\mathrm{B}}_{\mathrm{d}}^{0}$ mixing is large, $\left|V_{\mathrm{td}}\right|$ is expected to be large, so if this prediction is correct $V_{\mathrm{ub}}$ should soon be observable. The size of $C P$ violation is constrained to be at most about the observed value, though without further input we cannot determine the phases of the elements of the CKM matrix $[4,5]$.

In the next section we specify a possible structure of the theory, and write the neutral and charged currents. After that we examine several implications. We believe the general framework we are discussing may transcend particular implementations in models, or applications that require further input. Consequently, we list a number of model-dependent remarks only in the last section. We are presently studying models that realize our framework, and examining ways to constrain parameters. We also remark briefly in the last section on other approaches to fermion masses that consider the $\mathrm{u}-\mathrm{d}$ inversion.

Fermion masses. We assume the fermion mass matrix from the fundamental lagrangian is

$M_{\mathrm{u}}=\overline{\mathrm{u}}_{\mathrm{L}}\left(\begin{array}{ccc}m_{\mathrm{u}}^{0} & & 0 \\ & m_{\mathrm{c}}^{0} & \\ 0 & & m_{\mathrm{t}}^{0}\end{array}\right)$,

for the up-quarks, where rows and columns are labeled on the left and top, and

$M_{\mathrm{d}}={ }_{{ }_{\mathrm{L}}} \overline{\mathrm{D}}_{\mathrm{L}}\left(\begin{array}{ccccc}m_{\mathrm{d}}^{0} & & 0 & m_{1}^{\prime} \\ & m_{\mathrm{s}}^{0} & & m_{2}^{\prime} \\ 0 & & m_{\mathrm{b}}^{0} & m_{3}^{\prime} \\ m_{1} & m_{2} & m_{3} & M\end{array}\right)$,

for the down-quarks. That is, there exists another down-type quark that mixes with $\mathrm{d}, \mathrm{s}, \mathrm{b}$. If more down-type quarks also mix the form of $M_{\mathrm{d}}$ can be extended. We assume that $m_{d}^{0} \ll m_{\mathrm{u}}^{0}$; if a specific value were required, we could use $m_{\mathrm{d}}^{0} / m_{\mathrm{u}}^{0} \simeq m_{\mathrm{s}}^{0} / m_{\mathrm{c}}^{0} . \mathrm{D}_{\mathrm{L}}$ and $\mathrm{D}_{\mathrm{R}}$ are both $\mathrm{SU}(2)$ singlets; such a particle is referred to as vector-like.

Since no mixing occurs for the up-type quarks, we take $m_{\mathrm{u}}^{0}, m_{\mathrm{c}}^{0}, m_{\mathrm{t}}^{0}$ to be the measured masses $m_{\mathrm{u}}, m_{\mathrm{c}}, m_{\mathrm{t}}$ ( $m_{\mathrm{t}}$ will presumably be measured at the Tevatron collider if not before). For our purposes here we do not discuss the mixing effects on $m_{\mathrm{s}}^{0}$ and $m_{\mathrm{b}}^{0}$, so $m_{\mathrm{s}}^{0} \simeq m_{\mathrm{s}}$ and $m_{\mathrm{b}}^{0} \simeq m_{\mathrm{b}}$. All such effects will be discussed in ref. [6]. Only $m_{\mathrm{d}}^{0}$ is qualitatively modified in value.

$D_{\mathrm{L}}$ and $\mathrm{D}_{\mathrm{R}}$ are assumed to be $\mathrm{SU}(2)$ singlets, so $M$ is an allowed mass term $(\Delta I=0)$ and can be as large as allowed by the symmetries that operate. We assume $M$ is of the order of the electroweak scale, i.e. $M \sim M_{\mathrm{Z}}$ to within factors of two. The masses $m_{0}^{0\left({ }^{\prime}\right)}$ require SU(2) breaking $(\Delta I=1 / 2)$ so they are naively expected to be suppressed relative to $M$; assume $m_{i}^{\prime} \sim 1 \mathrm{GeV}$ (to within factors of two). The scale of $m_{i}$ is more subtle to assign. Naively we might expect $m_{i} \sim 1 \mathrm{GeV}$ also, since mixing is involved. On the other hand, no SU(2) breaking is required for $m_{i}$ (it is $\Delta I=0$ ) so $m_{i} \sim M$ could occur. In fact, models can be constructed either way that achieve our goals. In this paper we will concentrate on the case where $m_{i} \sim m_{i}^{\prime}$, and in ref. [6] several alternative models will be examined.

Eq. (2) can be diagonalized exactly, but the answer is not instructive. We only give expressions as an expansion in $m_{i} / M, m_{i}^{\prime} / M$. The diagonal down-quark mass matrix is 
$\left(\begin{array}{cccc}m_{\mathrm{d}} & & & 0 \\ & m_{\mathrm{s}} & & \\ & & m_{\mathrm{b}} & \\ 0 & & & m_{\mathrm{D}}\end{array}\right)=U_{\mathrm{L}}^{\mathrm{d}} M_{\mathrm{d}} U_{\mathrm{R}}^{\mathrm{d} \dagger}$,

with eigenvalues

$m_{\mathrm{d}} \simeq-m_{\mathrm{l}}^{\prime} m_{1} / M, \quad m_{\mathrm{s}} \simeq m_{\mathrm{s}}^{0}, \quad m_{\mathrm{b}} \simeq m_{\mathrm{b}}^{0}, \quad m_{\mathrm{D}} \simeq M$.

The diagonalizing matrices are

$$
\begin{aligned}
& U_{\mathrm{L}}^{\mathrm{d}}= \\
& \qquad \begin{array}{cc}
1 & \mu_{12} / m_{\mathrm{s}} \\
-\mu_{12}^{*} / m_{\mathrm{s}} & 1 \\
-\mu_{13}^{*} / m_{\mathrm{b}} & {\left[-m_{\mathrm{b}} /\left(m_{\mathrm{b}}^{2}-m_{\mathrm{s}}^{2}\right)\right]\left[\mu_{23}^{*}+\left(m_{\mathrm{s}} / m_{\mathrm{b}}\right) \mu_{32}\right]} \\
m_{1}^{\prime *} / M & m_{2}^{\prime *} / M
\end{array}
\end{aligned}
$$$$
\left.\begin{array}{cc}
\mu_{13} / m_{\mathrm{b}} & -m_{1}^{\prime} / M \\
{\left[m_{\mathrm{b}} /\left(m_{\mathrm{b}}^{2}-m_{\mathrm{s}}^{2}\right)\right]\left[\mu_{23}+\left(m_{\mathrm{s}} / m_{\mathrm{b}}\right) \mu_{32}^{*}\right]} & -m_{2}^{\prime} / M \\
1 & -m_{3}^{\prime} / M \\
m_{3}^{\prime *} / M & 1
\end{array}\right)
$$

where $\mu_{i j}=m_{i}^{\prime} m_{j} / M$, and

$U_{\mathrm{R}}^{\mathrm{d}}=U_{\mathrm{L}}^{\mathrm{d}}\left(m_{i} \leftrightarrow m_{i}^{\prime *}\right)$.

The CKM matrix is then the hermitian conjugate of the $3 \times 3$ block of $U_{\mathrm{L}}^{\mathrm{d}}$. The elements of ( $5 \mathrm{a}$ ) are at most linear in the two types of small parameters $\mu, m^{\prime} / M$. We must wonder also, however, if small effects or very small parameters get sizeable corrections at next order. In particular small flavour-changing neutral-current effects (FCNC) come to next order and the relation $\left|\left(U_{\mathrm{L}}^{\mathrm{d}}\right)_{31}\right|=\left|\left(U_{\mathrm{L}}^{\mathrm{d}}\right)_{13}\right|$ involves small numbers. In both cases the most important corrections result from the next order terms in $m^{\prime} / M$ [4]:

$\left(U_{\mathrm{L}}^{\mathrm{d}}\right)_{i j} \rightarrow\left(U_{\mathrm{L}}^{\mathrm{d}}\right)_{i j}+\left(m_{i}^{\prime} m_{j}^{\prime *} / M^{2}\right) \tilde{m}_{i}^{2} /\left[(-)^{\delta_{i j}} \tilde{m}_{j}^{2}-\tilde{m}_{i}^{2}\right]$,

where $i, j=1,2,3$ and $\tilde{m}$ stands for the observed masses $m_{\mathrm{d}, \mathrm{s}, \mathrm{b}}$. These terms are at most a few per cent of the previous matrix elements. The antihermiticity of $U_{\mathrm{L}}^{\mathrm{d}}$ (implying for instance equal moduli for symmetric matrix elements) follows from the fact that in this framework $U_{\mathrm{L}}^{\mathrm{d}}$ is, in agreement with experiment, infinitesimal. In other words, for small mixing unitarity requires the small off-diagonal elements be equal. In the CKM matrix small off-diagonal entries correspond to small angles in the Maiani parametrization [3]. However, in the Kobayashi-Maskawa parametrization [3] small rotations do not correspond to small (Euler) angles $\left(\theta_{1} \sim 0\right.$, whereas, up to a $C P$ phase, $\theta_{2} \sim-\theta_{3}$, but arbitrary and in general large), then being a less convenient parametrization for present applications. As it is well known, of the different parameters defining the model only four different functions (including a phase) can enter the CKM matrix (see eq. (5)).

Define

$$
\begin{aligned}
& A_{i \alpha}=\left(\begin{array}{cccc}
1 & -\mu_{12} / m_{\mathrm{s}} & -\mu_{13} / m_{\mathrm{b}} & m_{\mathrm{l}}^{\prime} / M \\
\mu_{12}^{*} / m_{\mathrm{s}} & 1 & {\left[-m_{\mathrm{b}} /\left(m_{\mathrm{b}}^{2}-m_{\mathrm{s}}^{2}\right)\right]\left[\mu_{23}+\left(m_{\mathrm{s}} / m_{\mathrm{b}}\right) \mu_{32}^{*}\right]} & m_{2}^{\prime} / M \\
\mu_{13}^{*} / m_{\mathrm{b}} & {\left[m_{\mathrm{b}} /\left(m_{\mathrm{b}}^{2}-m_{\mathrm{s}}^{2}\right)\right]\left[\mu_{23}^{*}+\left(m_{\mathrm{s}} / m_{\mathrm{b}}\right) \mu_{32}\right]} & 1 & m_{2}^{\prime} / M
\end{array}\right) \\
& =\left(V_{i j} \mid m_{i}^{\prime} / M\right) \text {, }
\end{aligned}
$$

with $i, j=1,2,3$ and $\alpha=1,2,3,4$. The usual CKM matrix is the $3 \times 3$ block of $A, V_{i j}$. Letting $\mathrm{u}_{i}, \mathrm{~d}_{\alpha}$ stand for the mass eigenstates, and

$$
B_{\alpha \beta}=\sum_{k=1,2,3}\left(U_{\mathrm{L}}^{\mathrm{d}}\right)_{\alpha k}\left(U_{\mathrm{L}}^{\mathrm{d} \dagger}\right)_{k \beta} \simeq\left(\begin{array}{cc}
1_{3 \times 3} & m_{i}^{\prime} / M \\
m_{j}^{* *} / M & \sum_{k=1,2,3}\left|m_{k}^{\prime}\right|^{2} / M^{2}
\end{array}\right)-\left(\begin{array}{cc}
m_{i}^{\prime} m_{j}^{\prime *} / M^{2} & 0 \\
0 & 0
\end{array}\right),
$$


the lagrangian is [7]

$L=\left(g_{2} / \sqrt{2}\right) \mathrm{W}_{\mu}^{\dagger} \overline{\mathrm{u}}_{\mathrm{L} i} \gamma^{\mu} \mathrm{d}_{\mathrm{L} \alpha} A_{i \alpha}+$ h.c. $+\frac{1}{2} \sqrt{g_{1}^{2}+g_{2}^{2}} \mathrm{Z}_{\mu}\left(\overline{\mathrm{u}}_{\mathrm{L} i} \gamma^{\mu} \mathrm{u}_{\mathrm{L} i}-\overline{\mathrm{d}}_{\mathrm{L} \alpha} \gamma_{\mu} \mathrm{d}_{\mathrm{L} \beta} B_{\alpha \beta}-2 \sin ^{2} \theta_{\mathrm{W}} J_{\mathrm{EM}}\right)$.

Because the off-diagonal elements of $B_{i j}$ are of order $m_{i}^{\prime} m_{j}^{*} / M^{2}, \mathrm{FCNC}$ of that order will arise, i.e. $B_{\mathrm{ds}}=-m_{1}^{\prime} m_{2}^{\prime *} / M^{2}$ [8]. Then strongest constraint is expected to arise from $\mathrm{K} \rightarrow \mu^{+} \mu^{-}$, which requires $\left|B_{\mathrm{ds}}\right|<2 \times 10^{-5}$. Given that each element $m_{i}^{\prime} / M \sim 10^{-2}$, there will be no trouble satisfying this constraint. Indeed, similar ratios are required for $m_{\mathrm{d}} \simeq 10 \mathrm{MeV} \simeq m_{1}^{\prime} m_{1} / M$, and to satisfy all constraints. Many other interesting FCNC will occur.

Further implications. The one clear prediction for the models we consider is that $\left|V_{\mathrm{td}}\right|=\left|V_{\mathrm{ub}}\right|$. As remarked earlier, this will be tested soon, since $\left|V_{\mathrm{td}}\right|$ is expected to be rather large because of the $\mathbf{B}_{\mathrm{d}}^{0}-\overline{\mathbf{B}}_{\mathrm{d}}^{0}$ mixing, while $V_{\mathrm{ub}}$ has not yet been observed. Other consequences depend on the details of values for $m_{i}, m_{i}^{\prime}$. For example, if $m_{i}=m_{i}^{\prime}$ then

$V_{\mathrm{ub}} \simeq-\left(m_{\mathrm{d}} / m_{\mathrm{s}}\right) V_{\mathrm{cb}} / V_{\mathrm{us}} \simeq 0.013$,

while if $m_{2}=m_{3}, V_{\mathrm{ub}}=m_{\mathrm{s}} V_{\mathrm{us}} / m_{\mathrm{b}} \simeq 0.008$, etc. Various models will be examined in ref. [6]. Note the current limit on $\left|V_{\mathrm{ub}} / V_{\mathrm{cb}}\right|$ is about 0.19 .

The magnitude of $M$ also cannot be specified accurately without further assumptions. Given that $m_{\mathrm{d}}$, three independent elements of $V_{i j}$ (as well as $C P$ violation), and various FCNC constraints must all be given correctly, in essentially any real theoretical model all the relevant parameters will be determined. Before presenting specific models, we prefer to do a thorough, detailed study to see if any unique results emerge.

$C P$ violation can be mediated by charged and neutral currents (see (7), (8) and (9)). Both contributions are comparable and of the correct order [4,5]. In this case $C P$ violation can be implemented with only two families for in general charged and tree level FCNC can not be made real simultaneously.

The phenomenology of the quark $\mathrm{D}$ is well understood. It is produced dominantly from $\mathrm{gg} \rightarrow \mathrm{D} \overline{\mathrm{D}}$ at a hadron collider, since it is a color triplet. The cross sections are the same as for any color triplet of a given mass. Its decays are determined by the lagrangian of eq. (9), and contain some interesting modes such as $D \rightarrow d \mu^{+} \mu^{-}$, $\mathrm{D} \rightarrow \mathrm{de}^{+} \mathrm{e}^{-}, \mathrm{D} \rightarrow \mathrm{d} v \bar{v}$ in addition to the usual decays $\mathrm{D} \rightarrow \mathrm{u} \mu v$, etc. [9]. These FCNC decays are at the level of one per cent per lepton flavor. They will provide clear confirmation of the discovery of D if it exists. For $M_{\mathrm{D}}<125$ $\mathrm{GeV}$ it can be observed at the Tevatron collider. Above that range we will have to wait for a future collider such as SSC or a higher luminosity Tevatron. We do not know of any dramatic effect expected from D in loops since no SU (2) breaking is required to give it mass. It couples to the Higgs field through its mixing with other quarks. Moreover, since they will be extra suppressed by inverse powers of its mass, it cannot significantly affect the Higgs boson production cross section and/or rare decays.

\section{Comments.}

(a) We are aware, of course, that this approach can be given an $\mathrm{E}_{6}$ interpretation [10] ${ }^{\# 1}$, making it attractive to superstring enthusiasts. The fundamental $\mathrm{E}_{6}$ representation, $27=16+10+1$, decomposed into $\mathrm{SO}(10)$ representations. The $S O(10)$ spinor representation, 16, contains $d_{L}, d_{R}$ usual. For the example we emphasized, with $m_{i} \ll M$, the $\mathrm{D}_{\mathrm{L}}, \mathrm{D}_{\mathrm{R}}$ are quark singlets in the 10 of $\mathrm{SO}(10)$. It is also possible to construct models with $\mathrm{D}_{\mathrm{L}, \mathrm{R}}$ as a $\mathrm{d}_{\mathrm{R}, \overline{\mathrm{R}}}$ component of a $27+\overline{27}$ when $m_{i} \simeq M$.

(b) A superstring-inspired $\mathrm{E}_{6}$ model lagrangian can be written to implement the example we have studied. In the simplest case the various masses arise from sneutrino vacuum expectation values, so $R$-parity is broken [12]. We believe no contradiction with experiment arises in such a model, and we are carefully examining the various numerical constraints. However, we emphasize that our basic approach should not be identified with

*1 For an introduction to $E_{6}$-superstring inspired models, see ref. [11]. 
a particular model at this stage. The value of a model is to help being sure that the approach is sensible; nature may choose a more clever implementation.

(c) The systematics of previous mass matrix studies [13] can be combined with our approach to reduce parameters. When such assumptions are added, generally a "prediction" for $m_{\mathrm{t}}$ will occur. Since $V_{\mathrm{td}}$ is also predicted, $\mathbf{B}_{\mathbf{d}}^{0}-\overline{\mathbf{B}}_{\mathrm{d}}^{0}$ mixing will strongly constrain such studies.

(d) One approach to understanding the $\mathrm{u}-\mathrm{d}$ ratio has been to assure that coupling constant renormalization effects from the GUT scale down to $m_{\mathrm{u}}, m_{\mathrm{d}}$, usually mainly affect $m_{\mathrm{u}}$ and account for the observed masses. However, in supersymmetric theories there are generally extra suppression factors $\sim M_{\mathrm{s}} / M_{\mathrm{G}}$ ( $M_{\mathrm{s}}$ is the scale where supersymmetry is broken and $M_{\mathrm{G}}$ the GUT scale). This extra factor is very small and renders negligible the needed effects of order $\alpha M_{\mathrm{w}}$. There appear to be exceptions, however [14].

(e) The matrices $U$ are anti-hermitean to the order we have written them. That is actually general, since a unitary matrix near the identity is anti-hermitean to lowest order. That is why the usual CKM matrix of moduli is almost symmetric, given that the mixings are small and unitarity is imposed.

(f) Note that $m_{1}^{(\prime)}$ cannot be zero since the corresponding diagonal element would then be $m_{\mathrm{d}}^{0}=m_{\mathrm{d}}<$ $m_{\mathrm{u}}^{0}=m_{\mathrm{u}}$. That is a strong constraint on models.

(g) A number of people have studied d-D mixing in the $E_{6}$ framework, mainly to check that no inconsistency appeared that would interfere with other aspects of the $E_{6}$ phenomenology' [15]. As far as we are aware, others have not taken as a primary point the presence of the $\mathrm{d}-\mathrm{D}$ mixing (without $\mathbf{u}$ mixing) that uncouples $m_{\mathrm{d}}$ from its unmixed value, and relates it to the mixing mass and to $m_{\mathrm{D}}$. It should be possible to test whether nature has used this mechanism, regardless of the connection to $E_{6}$ or other models, through the constraints on the CKM matrix and perhaps through the discovery of $D$. In the framework of left-right models neutrino masses seem also to require heavy D quarks [16].

\section{References}

[1] S.L. Glashow, Nucl. Phys. 22 (1961) 579;

A. Salam, in: Elementary particle theory, ed., N. Svartholm (Almqvist and Forlag, Stockholm, 1968);

S. Weinberg, Phys. Rev. Lett. 19 (1967) 1264.

[2] S.M. Barr, Phys. Rev. D 21 (1980) 1424;

R. Barbieri and D.V. Nanopoulos, Phys. Lett. B 91 (1980) 369; B 95 (1980) 43;

R. Barbieri, D.V. Nanopoulos and A. Masiero, Phys. Lett. B 104 (1981) 194;

R. Barbieri, D.V. Nanopoulos and D. Wyler, Phys. Lett. B 103 (1981) 303;

S.M. Barr, Phys. Rev. D 24 (1981) 1895;

M. Bowick and P. Ramond, Phys. Lett. B 103 (1981) 338;

Y. Achiman, Phys. Lett. B 131 (1983) 362;

F. Giuliani and F. Strocchi, Phys. Lett. B 154 (1985) 48; B 177 (1986) 82.

[3] N. Cabibbo, Phys. Rev. Lett. 10 (1963) 531;

M. Kobayashi and T. Maskawa, Progr. Theor. Phys. 49 (1973) 652;

L. Maiani, Proc. 1977 Symp. on Lepton and photon interactions (Hamburg, 1977).

[4] F. del Aguila and J. Cortés, Phys. Lett. B 156 (1985) 243; Nucl. Phys. B 271 (1986) 61.

[5] B. Mukhopadhyaya, A. Ray and A. Raychaudhuri, Phys. Lett. B 186 (1987) 147.

[6] F. del Aguila, G.L. Kane, M. Quirós and J. Vidal, in preparation.

[7] G.L. Kane and M. Peskin, Nucl. Phys B 195 (1982) 29.

[8] F. del Aguila and M.J. Bowick, Nucl. Phys. B 224 (1983) 107.

[9] F. del Aguila, E. Laermann and P. Zerwas, UF preprint UFTP-87-11 (1987).

[10] F. Gürsey, P. Ramond and P. Sikivie, Phys. Lett. B 60 (1976) 177;

Y. Achiman and B. Stech. Phys. Lett. B 77 (1978) 389;

Q. Shafi, Phys. Lett. B 79 (1978) 301;

H. Ruegg and T. Schucker, Nucl. Phys. B. 161 (1979) 388.

[11] M. Green, J. Schwarz and E. Witten, Superstring theory (Cambridge U.P., Cambridge, 1987), and references therein.

[12] J. Ellis, G. Gelmini, C. Jarlskog, G.G. Ross and J.W.F. Valle, Phys. Lett. B 150 (1985) 142;

J.W.F. Valle, Phys. Lett. B 186 (1987) 73. 
[13] H. Fritzsch, Phys. Lett. B 73 (1978) 317; Nucl. Phys. B 155 (1979) 189;

H. Georgi and D.V. Nanopoulos, Nucl. Phys. 155 (1979) 52;

B. Stech, Phys. Lett. B 130 (1983) 189;

G. Ecker, Z. Phys. C 24 (1984) 353.

[14] L.E.Ibáñez, Phys. Lett. B 117 (1982) 403;

S.M. Barr, Phys. Rev. D 31 (1985) 2979;

Y. Achiman, Phys. Lett. B 187 (1987) 309.

[15] J. Rosner, Comm. Nucl. Part. Phys. 15 (1986) 195;

R.W. Robinett, Phys. Rev. D 33 (1986) 1908;

V. Barger, N. Deshpande, R.J.N. Phillips and K. Whisnant, Phys. Rev. D 33 (1986) 1912.

[16] D. Chang and R.N. Mohapatra, Phys. Rev. Lett. 58 (1987) 1600. 